\title{
Confinement in two-dimensional QCD and the infinitely long pion
}

\author{
Colin M. Weller@ and Gerald A. Miller@ \\ University of Washington, Seattle Seattle, Washington 98195-1560, USA
}

(Received 6 December 2021; accepted 19 January 2022; published 17 February 2022)

Three current models of QCD in $(1+1)$ dimensions are examined and extended in light-front coordinates. A pion of high momentum is found to have an infinite extent along its direction of motion.

DOI: 10.1103/PhysRevD.105.036009

\section{INTRODUCTION}

There has been a recent revival of interest in theories involving two-dimensional (one-space-one time) treatments of QCD [1-4]. This stems from the need to include the effects of nonvanishing quark masses and also to enlarge the number of space-time variables of light-front holographic QCD to four. In the original treatments (see the review [5]) the chiral limit is used and the longitudinal light-front momentum fraction, $x$, is frozen [6], so that effectively the only degrees of freedom are light-front time and transverse position. The first effort aimed at including the effects of mass was contained in Ref. [7]. The present paper aims to unify the earlier treatments and to exhibit the confining aspects of the approaches in three-spatial dimensions using the spatial coordinate, $\tilde{z}$, that is canonically conjugate to the variable $x$ [8].

We begin by briefly summarizing light-front holographic QCD (LFHQCD) following [5]. Light-front (LF) quantization is a relativistic, frame-independent approach to describing the constituent structure of hadrons. The simple structure of the light-front vacuum allows an unambiguous definition of the partonic content of a hadron in QCD and of hadronic light-front wave functions. The QCD light-front Hamiltonian is constructed from the energy-momentum tensor of QCD. The spectrum and light-front wave functions of relativistic bound states are obtained from the resulting eigenvalue equation, an infinite set of coupled integral equations for the LF components in a complete basis of noninteracting $n$-particle states. This provides a quantum-mechanical probabilistic interpretation of the structure of hadronic states in terms of their constituents at the same light-front time $x^{+}=x^{0}+x^{3}$.

To a first semiclassical approximation, where quantum loops and quark masses are not included, the relativistic

Published by the American Physical Society under the terms of the Creative Commons Attribution 4.0 International license. Further distribution of this work must maintain attribution to the author(s) and the published article's title, journal citation, and DOI. Funded by SCOAP ${ }^{3}$. bound-state equation for light hadrons can be reduced to an effective LF Schrdinger equation. In conjugate coordinate space, the relevant dynamical variable is an invariant impact kinematical variable $\zeta=\mathbf{b} \sqrt{x(1-x)}$, where $\mathbf{b}$ is the transverse separation of the constituents. (Boldface notation specifies vectors of the two-dimensional transverse space.) The complexities of the strong interaction dynamics are then hidden in an effective potential $U(\zeta)$. Remarkably, the resulting light-front Hamiltonian has a structure that matches exactly the eigenvalue equations in anti-de Sitter (AdS) space [5]. This offers the possibility to explicitly connect the AdS wave function $\Phi(z)$ to the internal constituent structure of hadrons. Moreover, one can obtain the AdS wave equations by starting from the semiclassical approximation to light-front QCD in physical spacetime. This connection yields a relation between the coordinate $z$ of AdS space with the impact LF variable $\zeta$, thus giving the holographic variable $z$ a precise definition and intuitive meaning in light-front QCD [9].

\section{FORMALISM}

An effective light-front Schrödinger equation for the quark $\left(m_{1}\right)$-antiquark $\left(m_{2}\right)$, wave function $\psi(x, \mathbf{k})$, of a meson is given, for example, in [5]:

$$
\left[\frac{m_{1}^{2}}{x}+\frac{m_{2}^{2}}{1-x}+\frac{\mathbf{k}^{2}}{x(1-x)}+V_{\mathrm{eff}}\right] \psi=M_{h}^{2} \psi
$$

where $\mathbf{k}$ is the transverse relative momentum, the first three terms represent the kinetic energy in the center of momentum frame, $M_{h}$ is the invariant mass of the hadron, and $V_{\text {eff }}$ is an effective potential that acts in three-dimensional space. For the models used here $m_{1,2}$ are current quark masses.

Note that the kinetic energy term depends only on the two-dimensional vector $\mathbf{k} / \sqrt{x(1-x)}$, if the chiral limit $\left(m_{1,2}=0\right)$ is taken. Then, using coordinate space, and taking $V_{\text {eff }}$ to depend on $\zeta, V_{\text {eff }}=U_{\perp}(\zeta)$ one obtains 


$$
\left(-\frac{d^{2}}{d \zeta^{2}}+\frac{L^{2}}{\zeta^{2}}+U_{\perp}(\zeta)\right) \varphi(\zeta)=M^{2} \varphi(\zeta)
$$

which is the same as the equation of motion in the soft-wall AdS model [10] if $L$ is taken to be angular momentum, $\zeta$ is identified with the fifth dimension $z$ in AdS space, and the wave function $\varphi(\zeta)$ is identified with the string modes $\Phi(z)$.

Assuming duality with $\mathrm{AdS}_{5}$ suggests models for $U_{\perp}(\zeta)$, through a correspondence between the transverse Schrödinger equation (1) and the equation of motion for a spin- $J$ field in $\mathrm{AdS}_{5}$ [5]. For the soft-wall model [10], the effective potential reduces to an oscillator potential $U_{\perp}(\zeta)=\kappa^{4} \zeta^{2}+2 \kappa^{2}(J-1)$, where $\kappa$ is the strength of the holographic confinement and $J$ is the total angular momentum. For this potential, the spectrum of masses is $M_{\perp}^{2}=4 \kappa^{2}\left(n+\left(J+\left|L_{z}\right|\right) / 2\right)$, with $n$ the radial quantum number, and the transverse wave functions are the twodimensional oscillator eigenfunctions. The spectrum of the model provides for a linear Regge trajectory and a good fit to light meson masses. Using this potential leads to a massless pion $\left(n=0, L_{z}=0, J=0\right)$ in the chiral limit.

In Eq. (2) the variable $x$ is held fixed, leading to a $(2+1)$ space-time description that provides an excellent representation of the hadronic spectrum, but is manifestly incomplete because of the missing dynamics of the longitudinal direction. One incorporates [1-4,7] these dynamics by asserting that the total potential $V_{\text {eff }}$ of Eq. (1) is the sum of two terms: $V_{\text {eff }}=U_{\perp}(\zeta)+V_{\|}(x)$, with

$$
\left[\frac{m_{1}^{2}}{x}+\frac{m_{2}^{2}}{1-x}+V_{\|}\right] X_{n}(x)=M_{\|}^{2} X_{n}(x) .
$$

Under these assumptions the full wave function $\psi$ is given by the product

$$
\psi(x, \mathbf{b})=\varphi(\zeta) X_{n}(x)
$$

and $M_{h}^{2}=M_{\perp}^{2}+M_{\|}^{2}$. Here the normalization convention [11]

$$
\int_{0}^{1} \frac{\left|X_{n}(x)\right|^{2}}{x(1-x)} d x=1
$$

is used. It is helpful to define $\chi_{n}(x)=X_{n}(x) / \sqrt{x(1-x)}$.

One expects that the correct QCD-potential is not a sum of two independent terms. In that case the space of product wave functions forms a useful, complete, relativistic basis, such as advocated in [7] and implemented in [12]. Nevertheless, Refs. [1-4] compare the resulting values of $M_{h}^{2}$ with measured mesonic spectra.

Next, we consider the equation of the form $H_{\|} \chi_{n}=$ $M_{n}^{2} \chi_{n}$, with $H_{\|}$Hermitian. This is the form of the wave equation used by both 't Hooft and Callen, Coote and Gross [11] (and many others). Note that the $V_{\|}$of Refs. [2,13] are not diagonal in $x$. This is because confining potentials must have an explicit dependence on the coordinate-space variable, $\tilde{z}$, that is canonically conjugate to $x$. We shall see that none of the models of $V_{\|}$in current use is of the form $V_{\|}(x)$.

With the fundamental longitudinal wave equation the usual orthonormal equation applies

$$
\int d x \chi_{n}^{*}(x) \chi_{m}(x)=\delta_{n m},
$$

as explicitly stated in Ref. [11]. This form is general once the Hermiticity of $H_{\|}$is specified such as in $[1,2,13]$. Next, consider the matrix element.

$$
\langle n|H| m\rangle=\int d x d y \chi_{n}^{*}(x) H(x, y) \chi_{m}(y) .
$$

The Hamiltonian can be expressed (for an equivalent quark and antiquark mass $\left.m_{1}\right)$ as $H(x, y)=\frac{m_{1}^{2}}{x(1-x)} \delta(x-y)+$ $V_{\|}(x, y)$, so that

$$
\begin{aligned}
\langle n|H| m\rangle= & \int d x \frac{m^{2}}{x(1-x)} \chi_{n}^{*}(x) \chi_{m}(x) \\
& +\int d x d y \chi_{n}^{*}(x) V_{\|}(x, y) \chi_{m}(y) .
\end{aligned}
$$

The longitudinal potential $V_{\|}$must be chosen to determine the function $\chi_{n}(x)$. There are two choices in the literature. The first two Li-Vary (LV) [2] and the 't Hooft model (tH) [13], are given by

$$
\begin{aligned}
V_{\mathrm{LV}}(x) \chi_{n}(x) & =-\sigma^{2} \partial_{x} x(1-x) \partial_{x} \chi_{n}(x) \\
\left(V_{\mathrm{tH}} \chi_{n}\right)(x) & =\frac{g^{2}}{\pi} P \int_{0}^{1} d y \frac{\chi_{n}(x)-\chi_{n}(y)}{(x-y)^{2}},
\end{aligned}
$$

with the principal value is defined [14] as $P \frac{f(x, y)}{(x-y)^{2}} \equiv$ $\frac{1}{2}\left[\frac{f(x, y)}{(x-y+i \epsilon)^{2}}+\frac{f(x, y)}{(x-y-i \epsilon)^{2}}\right]$ in the limit $\epsilon \rightarrow 0$. The function $V_{\mathrm{LV}}(x)$ is used in [2], and has the advantage that exact solutions are available in terms of Jacobi polynomials. The 't Hooft model [13], obtained in the large- $N$ limit of twodimensional QCD, is used in $[3,4,7]$. This is the natural choice for a confining potential in one spatial dimension. When QCD is quantized in the light-cone gauge, such a potential appears automatically as an instantaneous Coulomb-like interaction, $V_{\mathrm{tH}}(\tilde{z})=g^{2}|\tilde{z}| e^{-\epsilon|\tilde{z}|}$, between quark currents [14], with $\tilde{z}$ as the longitudinal position operator [8] that is canonically conjugate to the longitudinal momentum variable $x$. Taking the Fourier transform of $V_{\mathrm{tH}}(\tilde{z})$ using the transformation $\langle x \mid \tilde{z}\rangle=e^{-i x \tilde{z}} / \sqrt{2 \pi}$ and including effects of the quark self-energy via the term $\chi(x)$ term of the principle-value integral leads to the expression 
appearing in Eq. (9). Note that in the 't Hooft model, the masses $m_{1,2}$ are explicitly current quark masses. The 't Hooft model was extensively studied during the 1970s; see the review [15].

The third approach [1] is to assert that $\chi(x)$ is a Gaussian in the invariant mass-squared: $\chi(x)=\mathcal{N} \exp \left[-1 /\left(2 \kappa^{2}\right) \times\right.$ $\left.\left(-m_{1}^{2} / x+m_{2}^{2} /(1-x)\right)\right]$, where $\mathcal{N}$ is a normalization constant [5]. This model is termed the invariant mass wave function (IMWF).

To obtain an expression for the Hamiltonian using the $X$ normalization: define $X_{n}(x) \equiv \sqrt{x(1-x)} \chi_{n}(x)$ so that

$\langle n|H| m\rangle=\int \frac{d x}{x(1-x)} \tilde{X}_{n}(x) \frac{m^{2}}{x(1-x)} X_{m}(x)+\left\langle n\left|V_{\|}\right| m\right\rangle$,

with

$$
\left\langle n\left|V_{\|}\right| m\right\rangle=\int \frac{d x d y}{x(1-x)} \tilde{X}_{n}(x) \frac{\sqrt{x(1-x)}}{\sqrt{y(1-y)}} V(x, y) X_{m}(y) .
$$

The effective potential, $\frac{\sqrt{x(1-x)}}{\sqrt{y(1-y)}} V(x, y)$ appearing between the two wave functions is not Hermitian because it is not symmetric in the variables $x$ and $y$. The function $\tilde{X}_{n}$ are obtained using the effective potential $\frac{\sqrt{y(1-y)}}{\sqrt{x(1-x)}} V(x, y)$.

The consequence of this lack of Hermiticity is that, for example, the resulting wave equation for the t' Hooft model would be of the form:

$$
\begin{aligned}
M_{n}^{2} X_{n}(x)= & \frac{m^{2}}{x(1-x)} X_{n}(x) \\
& -\frac{g^{2}}{\pi} \mathcal{P} \int d y \frac{\left(X_{n}(x)-\frac{\sqrt{x(1-x)}}{\sqrt{y(1-y)}} X_{n}(y)\right)}{(x-y)^{2}} .
\end{aligned}
$$

This equation was solved in Ref. [7]. The non-Hermitian nature of the effective potential arises from the $X$ normalization. Instead, the normalization given in Eq. (6) is used for the rest of the paper.

We now begin to discuss the differences between the two potentials of Eq. (9). In the chiral limit, the ground state wave function of both $[2,11]$ can be seen from the differential equation for $M_{\|}^{2}$ to be simply (dropping the subscript) $\chi(x)=1$, with an eigenvalue $M_{\|}^{2}=0$. Reference [1] also have $\chi(x)=1$ in the chiral limit.

The LFHQCD formalism [5] achieved excellent reproduction of the hadronic spectrum. Using $\chi(x)=1$ along with $M_{\|}^{2}=0$ preserves that ground-state spectrum.
The useful identity [11]:

$$
M_{\|}^{2} \int_{0}^{1} d x \chi(x)=\int_{0}^{1} d x \chi(x)\left[\frac{m_{1}^{2}}{x}+\frac{m_{2}^{2}}{1-x}\right]
$$

holds in both the Li-Vary and 't Hooft models. The need to have finite results for both sides of this equation signifies that $\chi(x)$ vanishes at the endpoints, $x=0,1$.

Given the similarities between the Li-Vary and 't Hooft models, it is natural to initially expect that any differences between the models might be thought to be small. This is far from the case. Using coordinate space helps in understanding that there indeed are significant differences between the potentials. This is done by evaluating $V_{\mathrm{LV}}(x)$ in coordinate space.

$$
\begin{aligned}
\left\langle\tilde{z}\left|V_{\mathrm{LV}}(x)\right| \tilde{z}^{\prime}\right\rangle & =\sigma^{2} \tilde{z} \tilde{z}^{\prime} \int_{0}^{1} \frac{d x}{2 \pi} e^{i x\left(\tilde{z}^{\prime}-\tilde{z}\right)} x(1-x) \\
& =\frac{\sigma^{2}}{2 \pi} \tilde{z} \tilde{z}^{\prime} e^{i \frac{\left(z^{\prime}-\tilde{z}\right)}{2}} \frac{j_{1}\left(\frac{\tilde{z}^{\prime}-\tilde{z}}{2}\right)}{\tilde{z}^{\prime}-\tilde{z}},
\end{aligned}
$$

where $j_{1}$ is a spherical Bessel function. The diagonal elements of this operator are given by $\sigma^{2} \tilde{z}^{2} / 6$ which is in stark contrast to the linear behavior of the potential in the ' $t$ Hooft model. Moreover, there is a significant coordinatespace nonlocality in $V_{\mathrm{LV}}(x)$ that does not occur for $V_{\mathrm{tH}}$. Another difference is that the potential $V_{\mathrm{LV}}(x)$ is complex although Hermitian.

The most salient difference between the two potentials is in the high-energy spectrum: $M_{\|}^{2}(\mathrm{tH}) \approx g^{2} k$, [11] where $k$ is an integer. The high energy spectrum of the 't Hooft model is much more compact than that of the Li-Vary model. For large values of $k, \chi_{\mathrm{tH}}(x)=\sqrt{2} \sin (\pi k x+\delta(k, x))$. The phase shift $\delta(k, x)$ is given in Ref. [14].

The spectrum corresponding to the Brodsky-de Teramond IMWF model [1] has not been provided before. The lore is that this model does not come from a wave equation. Here we develop a wave equation of the SturmLiouville form that does yield the IMWF and discuss the properties of the solutions. The first step is asserting that the IMWF is the solution of some wave equation. The presentation is simplified by using $m_{1,2}=m$ and also by employing the variable $y>0$, with $y^{2}=1 /(x(1-x))$. Then using $\phi(y)=e^{\frac{-m^{2} y^{2}}{2 k^{2}}}$, consider the differential equation:

$$
-\frac{d}{d y} f(y) \frac{d \phi(y)}{d y}+m^{2} y^{2} \phi(y)=M^{2} \phi(y),
$$

with $f(y)$ to be determined. The term $m^{2} y^{2}$ is the familiar kinetic energy term. Using the expression for $\phi(y)$ and equating the left- and right-sides of the equation leads to the results $f=\kappa^{4} / m^{2}$ and $M^{2}=\kappa^{2}$. A peculiar feature is that the ground-state mass is independent of $m$. The wave 
function that leads to the ground state IMWF is then given by

$$
-\frac{\kappa^{4}}{m^{2}} \phi^{\prime \prime}+m^{2} y^{2} \phi=M^{2} \phi,
$$

which is of a familiar harmonic oscillator form. This can be converted to an equation in the variable $x$ by using $\frac{d x}{d y}=$ $\frac{1}{x-1 / 2} x^{3 / 2}(1-x)^{3 / 2} \equiv g(x)$, so that Eq. (16) becomes

$$
-g(x) \frac{\kappa^{4}}{m^{2}} \frac{d}{d x} g(x) \frac{d \phi(x)}{d x}+\frac{m^{2} \phi(x)}{x(1-x)}=M^{2} \phi(x),
$$

which is of the Sturm-Liouville form for $0<x<1 / 2$ and $1 / 2<x<1$. The ground state energy is $\kappa^{2}$. Each excitation in energy increases $M^{2}$ by $2 \kappa^{2}$. The feature that the spectrum does not depend on quark masses does not seem reasonable and we therefore discard Eq. (16).

The next step is to compare two remaining approaches $[2,13]$ in the limit that the quark masses are nonzero, but small compared to the strength parameter of the model. For simplicity we remain with the case that $m_{1,2}=m$. The Li-Vary result is that

$$
M_{\|}^{2}(\mathrm{LV})=2 \sigma m+4 m^{2} .
$$

Their model uses the parameters $m=15 \mathrm{MeV}$, and $\sigma=620 \mathrm{MeV}$. 't Hooft found that the ground-state wave function $\chi(x)$ is well approximated by the form $\chi_{0}(x)=x^{\beta}(1-x)^{\beta}$. Analysis of the behavior for the solution of the wave equation for $x \rightarrow 0$ or 1 shows that $\beta$ should satisfy the transcendental equation $\frac{m^{2} \pi}{g^{2}}-1+\pi \beta \cot \pi \beta=0$. For small values of $m$, the quantity $\beta \pi$ must also be small so that $\beta=\sqrt{\frac{3}{\pi}} \frac{m}{g}$. Using $\chi_{0}$ on both sides of Eq. (13) yields that $M_{\|}^{2}=m^{2}\left(\frac{2}{\beta}+4\right)$, or equivalently

$$
M_{\|}^{2}(\mathrm{tH})=2 \sqrt{\frac{\pi}{3}} g m+4 m^{2} .
$$

Using the average value of the $u$ and $d$ quark masses, $m=3.5 \mathrm{MeV}$ [16] with $M_{L}^{2}=140 \mathrm{MeV}$, tells us that $g=2700 \mathrm{MeV}$, and $\beta=0.00126$. Thus, these two models contain a $(1+1)$-dimensional version of the Gell-MannOakes-Renner [17] relation in which the squared mass of the ground state is proportional to the current quark mass.

It is worthwhile to note that the use of current-quark masses in the 't Hooft model causes the two different models to obtain very different masses of the first excited state. Reference [2] obtains excitation energies of approximately $1 \mathrm{GeV}$ and associates these values with excited states of the pion. In the present work, using modern values of the current quark masses [16] gives the lowest excited state a mass on the order of $g$, or about $3 \mathrm{GeV}$. This is high enough into the continuum of states with large widths to be unobservable. Thus, the version of the 't Hooft model used here preserves the spectra produced by LFHQCD.

\section{COORDINATE SPACE TREATMENT}

The confining aspects of the 't Hooft model have been well-studied long ago $[11,13]$, using a momentum-space $(x)$ dependence approach based on studying the cancellation of infrared singularities. Another, possibly more intuitive approach, may be obtaining by examining coordinate-space wave functions that depend on the canonically conjugate spatial variable, $\tilde{z}$. An intuitive way to think about this variable is that it is the separation between the quark and antiquark in the direction of motion of a pion moving with high momentum.

Coordinate-space wave functions are obtained using the transformation

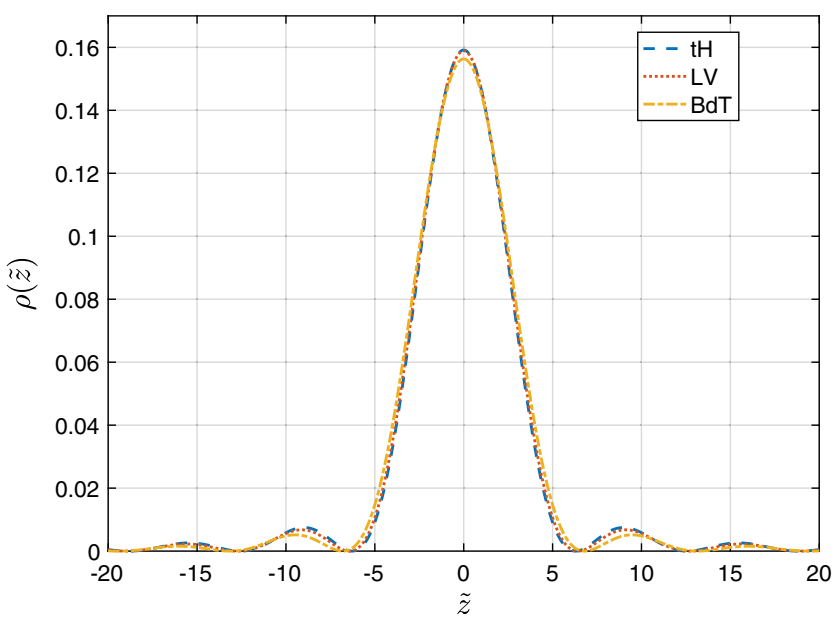

(a)

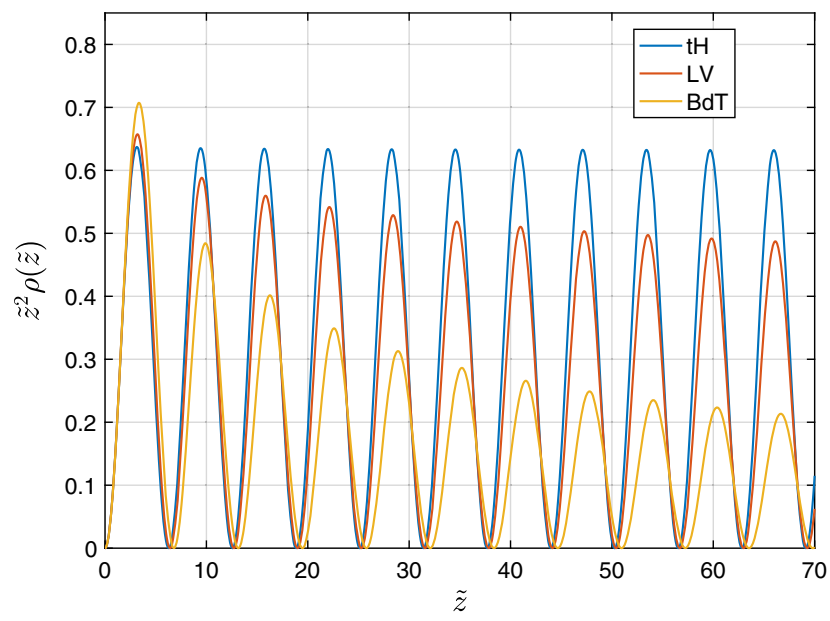

(b)

FIG. 1. Plots of the resulting density, $\rho(\tilde{z})$, and $\tilde{z}^{2} \rho(\tilde{z})$ are given for all three models. The discrepancy between the models in $\rho(\tilde{z})$ is small but finite, and the $1 / \tilde{z}^{2}$ asymptotic behavior is confirmed. 


$$
\chi(\tilde{z}) \equiv \int_{0}^{1} \frac{d x}{\sqrt{2 \pi}} e^{i x \tilde{z}} \chi(x)
$$

It is useful to examine the density $\rho(\tilde{z}) \equiv|\chi(\tilde{z})|^{2}$, a realvalued quantity, for the three models are shown in Fig. 1(a). These results seem very similar because of the relatively small quark masses of the three models. The densities seem to go to 0 for large absolute values of $\tilde{z}$. They do, but there in an interesting feature seen by plotting $\tilde{z}^{2} \rho(\tilde{z})$ in Fig. 1(b): the densities fall as $1 / \tilde{z}^{2}$.

This behavior may be understood by making a asymptotic expansion, obtained by using $e^{i \tilde{z} x}=1 /(i \tilde{z}) \partial_{x} e^{i \tilde{z} x}$ and the feature that $\chi(x)$ vanishes at the end points. Then

$$
\lim _{|\tilde{z}| \rightarrow \infty} \chi(\tilde{z})=\frac{i}{\tilde{z}} \int_{0}^{1} \frac{d x}{\sqrt{2 \pi}} e^{i x \tilde{z}} \partial_{x} \chi(x)
$$

Squaring this quantity leads to the stated $1 / \tilde{z}^{2}$ dependence.

The similarity of the behaviors for small masses suggests that the chiral limit should be examined. In this case, $\chi(x)=1$ for all three models. Then a simple closed-form expression for $\chi(\tilde{z})$ can be obtained. The resulting density, $\rho_{\chi}(\tilde{z})$ is given by

$$
\rho_{\chi}(\tilde{z})=\frac{2}{\pi} \frac{\sin ^{2} \tilde{z} / 2}{\tilde{z}^{2}},
$$

an expression that accounts explicitly for the oscillatory behavior as well as the $1 / \tilde{z}^{2}$ asymptotic behavior. One may also examine the spatial extent of the pion wave function by considering the mean-square value of $\tilde{z}^{2}$, that is given by the ground-state expectation value:

$$
\left\langle\chi\left|\tilde{z}^{2}\right| \chi\right\rangle=\frac{2}{\pi} \int_{-\infty}^{\infty} d \tilde{z} \sin ^{2}(\tilde{z} / 2)=\infty .
$$

In the chiral limit the pion has an infinite spatial extent, true for all three models.
It is necessary to see how or if this infinite size conflicts with current understanding. First note that the elastic pion form factor of LFHQCD has already been computed using $\chi(x)=1$; see e.g., Ref. [18]. The infinite extent is buried within the integrals needed to compute the elastic form factor, a consequence the ability to probe with only transverse momentum transfers; see e.g., the review [19].

The relevance of the infinite longitudinal extent can be understood in analogy with the familiar Ioffe-time argument [20-23] for deep inelastic scattering at small values of Bjorken $x$. The idea is that an incident virtual photon fluctuates into a $q \bar{q}$ pair. The energy difference, $\Delta E$, between the two states is very small, so that the fluctuation lives for a long time $\Delta t>1 / \Delta E$. For virtual photons of high energy, the extent of the fluctuation is $c \Delta t$ is very large. Now consider a high-momentum pion incident on a target. The value of $\Delta E$ goes to 0 if both the pion and its constituents are massless. Thus the infinite extent of a massless pion is part of standard lore.

\section{SUMMARY}

We summarize. The similarities and differences between the three models of Refs. [1,2,13] are exhibited. Very significant differences in the excitation spectra, at both low and high energies, are obtained, even though all of the ground-state wave functions are the same in the chiral limit. If the 't Hooft model is used along with current values of light-quark masses, the original spectrum calculations reviewed in Ref. [5] are preserved. Finally, the pion is shown to have an infinite extent in the longitudinal direction.

\section{ACKNOWLEDGMENTS}

This work was supported by the U.S. Department of Energy Office of Science, Office of Nuclear Physics under Award No. DE-FG02-97ER-41014. We thank J. Hiller, Y. Li, J. P. Vary, R. Sandapen, G. F. de Téramond and S. J. Brodsky for helpful comments.
[1] G. F. De Téramond and S. J. Brodsky, Longitudinal dynamics and chiral symmetry breaking in holographic light-front QCD, Phys. Rev. D 104, 116009 (2021).

[2] Y. Li and J. P. Vary, Light-front holography with chiral symmetry breaking, Phys. Lett. B 825, 136860 (2022).

[3] M. Ahmady, H. Dahiya, S. Kaur, C. Mondal, R. Sandapen, and N. Sharma, Extending light-front holographic QCD using the 't Hooft equation, Phys. Lett. B 823, 136754 (2021).

[4] M. Ahmady, S. L. MacKay, S. Kaur, C. Mondal, and R. Sandapen, Hadron spectroscopy using the light-front holographic Schrödinger equation and the 't Hooft equation, Phys. Rev. D 104, 074013 (2021).

[5] S. J. Brodsky, G. F. de Teramond, H. G. Dosch, and J. Erlich, Light-front holographic QCD and emerging confinement, Phys. Rep. 584, 1 (2015).

[6] A. B. Sheckler and G. A. Miller, Mystery of Bloom-Gilman duality: A light-front holographic QCD perspective, Phys. Rev. D 103, 096018 (2021).

[7] S. S. Chabysheva and J. R. Hiller, Dynamical model for longitudinal wave functions in light-front holographic QCD, Ann. Phys. (Amsterdam) 337, 143 (2013). 
[8] G. A. Miller and S. J. Brodsky, Frame-independent spatial coordinate $\tilde{z}$ : Implications for light-front wave functions, deep inelastic scattering, light-front holography, and lattice QCD calculations, Phys. Rev. C 102, 022201 (2020).

[9] S. J. Brodsky and G. F. de Teramond, Light-front holography and AdS/QCD: A first approximation to QCD, eCONF C0906083, 17 (2009).

[10] A. Karch, E. Katz, D. T. Son, and M. A. Stephanov, Linear confinement and AdS/QCD, Phys. Rev. D 74, 015005 (2006).

[11] C. G. Callan, Jr., N. Coote, and D. J. Gross, Two-dimensional Yang-Mills theory: A model of quark confinement, Phys. Rev. D 13, 1649 (1976).

[12] Y. Li, P. Maris, X. Zhao, and J. P. Vary, Heavy quarkonium in a holographic basis, Phys. Lett. B 758, 118 (2016).

[13] G. 't Hooft, A two-dimensional model for mesons, Nucl. Phys. B75, 461 (1974).

[14] R. C. Brower, W. L. Spence, and J. H. Weis, Bound states and asymptotic limits for quantum chromodynamics in two dimensions, Phys. Rev. D 19, 3024 (1979).

[15] J. R. Ellis, Applications of two-dimensional QCD, Acta Phys. Pol. B 8, 1019 (1977).
[16] P. A. Zyla et al. (Particle Data Group), Review of particle physics, Prog. Theor. Exp. Phys. 2020, 083C01 (2020).

[17] M. Gell-Mann, R. J. Oakes, and B. Renner, Behavior of current divergences under $\mathrm{SU}_{3}$ x $\mathrm{SU}_{3}$, Phys. Rev. 175, 2195 (1968).

[18] G. F. de Teramond, T. Liu, R. S. Sufian, H. G. Dosch, S. J. Brodsky, and A. Deur (HLFHS), Universality of Generalized Parton Distributions in Light-Front Holographic QCD, Phys. Rev. Lett. 120, 182001 (2018).

[19] G. A. Miller, Transverse charge densities, Annu. Rev. Nucl. Part. Sci. 60, 1 (2010).

[20] V. N. Gribov, B. L. Ioffe, and I. Ya. Pomeranchuk, What is the range of interactions at high-energies, Yad. Fiz. 2, 768 (1965).

[21] B. L. Ioffe, Space-time picture of photon and neutrino scattering and electroproduction cross-section asymptotics, Phys. Lett. 30B, 123 (1969).

[22] Y. V. Kovchegov and E. Levin, Quantum Chromodynamics at High Energy (Cambridge University Press, Cambridge, England, 2012), Vol. 33.

[23] V. Braun and D. Müller, Exclusive processes in position space and the pion distribution amplitude, Eur. Phys. J. C 55, 349 (2008). 